\title{
Eyelid sebaceous carcinoma: Validation of the 8th edition of the American Joint Committee on cancer T staging system and the prognostic factors for local recurrence, nodal metastasis, and survival
}

\author{
Yun Hsia ${ }^{1}$ - Ching-Yuan Yeh ${ }^{2} \cdot$ Yi-Hsuan Wei ${ }^{1}$ - Lily-Wei Chen ${ }^{3} \cdot$ Shu-Lang Liao $\mathbb{D}^{1,4}$
}

Received: 24 January 2018 / Revised: 5 March 2019 / Accepted: 5 March 2019 / Published online: 31 May 2019

(c) The Royal College of Ophthalmologists 2019

\section{Learning Objectives}

Upon completion of this activity, participants will be able to:

1. Describe clinicopathological features of eyelid SGC in the ethnic Chinese population in Taiwan, according to a retrospective study

2. Determine prognostic factors for eyelid SGC in the ethnic Chinese population in Taiwan, according to a retrospective study

3. Compare the 7th and 8th editions of the AJCC staging system for diagnostic performance regarding the outcomes of local recurrence, regional lymph node metastasis, and tumor-related death, according to a retrospective study

\section{Continuing Medical Education}

In support of improving patient care, this activity has been planned and implemented by Medscape, LLC and Springer Nature. Medscape, LLC is jointly accredited by the Accreditation Council for Continuing Medical Education (ACCME), the Accreditation Council for Pharmacy Education (ACPE), and the American Nurses Credentialing Center (ANCC), to provide continuing education for the healthcare team.

Medscape, LLC designates this Journal-based CME activity for a maximum of 1.00 AMA PRA Category 1 Credit(s). Physicians should claim only the credit commensurate with the extent of their participation in the activity.

Successful completion of this CME activity, which includes participation in the evaluation component, enables the participant to earn up to 1.0 MOC points in the American Board of Internal Medicine's (ABIM) Maintenance of Certification (MOC) program. Participants will earn MOC points equivalent to the amount of CME credits claimed for the activity. It is the CME activity provider's responsibility to submit participant completion information to ACCME for the purpose of granting ABIM MOC credit.

\section{Credit hours}

1.0

Release date: 31 May 2019

Expiration date: 31 May 2020

Post-test link: https://medscape.org/eye/posttest912117

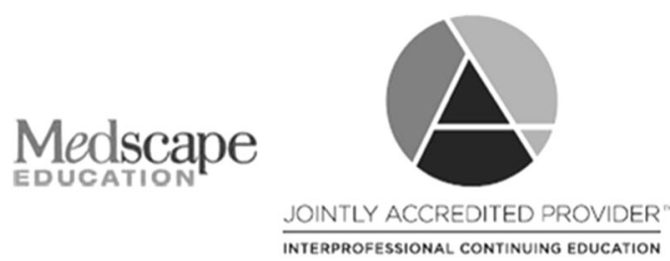

These authors contributed equally: Yi-Hsuan Wei, Shu-Lang Liao

Shu-Lang Liao

liaos189@ntu.edu.tw

1 Department of Ophthalmology, National Taiwan University Hospital, Taipei, Taiwan

2 Department of Internal Medicine, National Taiwan University Hospital, Taipei, Taiwan
3 University of Massachusetts Medical School, Worcester, MA, USA

4 College of Medicine, National Taiwan University, Taipei, Taiwan 


\title{
Authors/Editors disclosure information
}

S.S. has disclosed the following relevant financial relationships: Served as an advisor or consultant for: Allergan, Inc.; Bayer AG; Boehringer Ingelheim Pharmaceuticals, Inc.; Heidelberg Pharma GmbH; Novartis Pharmaceuticals Corporation; Optos; Roche. Served as a speaker or a member of a speakers bureau for: Allergan, Inc.; Bayer AG; Novartis Pharmaceuticals Corporation; Optos. Received grants for clinical research from: Allergan, Inc.; Bayer AG; Boehringer Ingelheim Pharmaceuticals, Inc.; Novartis Pharmaceuticals Corporation; Optos. Y.H., C.-Y.Y., Y.-H.W., L.-W.C., S.-L.L. have disclosed no relevant financial relationships.

\section{Journal CME author disclosure information}

Laurie Barclay has disclosed no relevant financial relationships.

\begin{abstract}
Purpose To investigate the clinicopathological features and prognostic factors for eyelid sebaceous gland carcinoma (SGC) in an ethnic Chinese population and to validate the performance of the T category of the 8th edition AJCC staging systems, with the aim of providing information for refinements.

Methods Sixty-three patients with pathological diagnosis of SGC were enroled retrospectively. The clinicopathological features, treatments, and outcomes were collected. Prognostic factors associated with the outcome of local recurrence, regional lymph node metastasis, and tumour-related death were analysed. The performance analysis was performed by comparing the predictive value for survival and the monotonicity of gradients between the 7 th and 8th staging systems.

Results The distribution of T1:T2:T3:T4 tumours according to the 7th and 8th edition was 6:40:16:1 and 23:26:5:9, respectively. Positive surgical margin was a poor prognostic factor. Local recurrence was associated with more aggressive histopathological features and surrounding structure invasions. Regional lymph node metastasis was associated with larger tumours. The $\mathrm{T}$ category of 8 th edition showed better predictability for local recurrence and regional lymph node metastasis, while the $\mathrm{T}$ category of 7 th edition had better monotonicity of gradients. Tumours classified as T2c or worse had higher risk of regional lymph node metastasis, while tumours T3b or worse in the 8th edition had more tumour-related death.

Conclusions Patients with higher T category are at risk of regional lymph node metastasis and tumour-related death. Further refinement of the T category of AJCC staging system can focus on the predictability for local recurrence and the monotonicity of gradients.
\end{abstract}

\section{Introduction}

Eyelid sebaceous gland carcinoma (SGC) is slow-growing but potentially lethal [1]. It is notorious for its varied clinical appearance and masquerades as benign conditions. The difficulties in making the diagnosis can lead to delays in treatment, resulting in great morbidity and mortality [2]. Although the data of absolute incidence in different ethnic groups is limited in the literatures, the rate of different eyelid cancers varies among different races. In the Caucasian population, basal cell carcinoma is the most frequently encountered and constitutes $85-95 \%$ of eyelid cancers [3], while SGC is relatively rare, accounting for 1-5.5\% [4]. SGC is much more common among Asians. Among eyelid malignancies, SGC was reported to account for $23.6 \%$, $28.9 \%, 29.3 \%$, and $32.6 \%$ in Taiwan, Japan, China, and India, respectively [4-7].

The prognostic factors for eyelid SGC have been discussed in previous studies, including canthal involvement, larger tumour diameter, and perivascular invasion [8]. However, due to the rarity of the disease, it is not clear if the prognostic factors may vary in different ethnic groups. In addition, eyelid SGC uses the same AJCC staging system as basal cell carcinoma, squamous cell carcinoma, and sweat gland carcinoma of eyelids. Therefore, it is important to validate the ability of the staging system for predicting patient prognosis for each type of eyelid malignancy. The predictability of outcomes for eyelid SGC based on the T category of the 7th edition American Joint Committee on Cancer (AJCC) staging system has been determined in Caucasian, Japanese, and Indian populations [9-11]. The 8th edition AJCC staging system was published in 2016. Evolving from the 7 th edition to 8th edition [12, 13], the subjective criteria (e.g. resectability) was abandoned, and the cut-off point of $\mathrm{T} 2$ tumour shifted from 5 to $10 \mathrm{~mm}$ (Table 1). These changes have a great impact on the distribution of patients across the stages; therefore, we aimed to examine the reliability of the new edition.

In the present study, we analysed the clinicopathological features and prognostic factors for eyelid SGC in the ethnic Chinese population in Taiwan. We also compared the 
Table 1 Comparison of the T category 7th and 8th editions AJCC staging system

\begin{tabular}{|c|c|c|c|c|}
\hline \multicolumn{2}{|c|}{$\begin{array}{l}7 \text { th } \\
\text { edition }\end{array}$} & \multicolumn{2}{|c|}{$\begin{array}{l}\text { 8th } \\
\text { edition }\end{array}$} & Major changes \\
\hline \multicolumn{4}{|c|}{ TX: Primary cancer cannot be assessed; T0: No evidence of primary tumour; Tis: Carcinoma in situ } & (1) The cut point of $\mathrm{T} 2$ tumour was shifted from 5 to $10 \mathrm{~mm}$ \\
\hline $\mathrm{T} 1$ & $\begin{array}{l}\text { Tumour } \leq 5 \mathrm{~mm} \text {; not invading tarsal } \\
\text { plate or eyelid margin }\end{array}$ & $\begin{array}{l}\text { T1a } \\
\text { T1b } \\
\text { T1c }\end{array}$ & $\begin{array}{l}\text { Tumour } \leq 10 \mathrm{~mm} \text { in greatest dimension } \\
\text { Tumour does not invade tarsal plate or eyelid margin } \\
\text { Tumour invade tarsal plate or eyelid margin } \\
\text { Tumour invade full thickness of the eyelid }\end{array}$ & $\begin{array}{l}\text { (2) Elimination of perineural invasion as a criterium for T3a } \\
\text { (3) T3b or T4 in the 7th edition was based on the resectability } \\
\text { of tumour which may be affected by the surgeon's } \\
\text { technique and facility. This has been changed to more } \\
\text { objective findings, tumour size, and orbital or facial } \\
\text { structure involvement } \\
\text { (4) Tumours } \leq 5 \mathrm{~mm} \text { with tarsal or full thickness involvement }\end{array}$ \\
\hline $\mathrm{T} 2 \mathrm{a}$ & $\begin{array}{l}\text { Tumour }>5 \mathrm{~mm} \text {, but not }>10 \mathrm{~mm} \text {, or } \\
\text { any tumour invading tarsal plate or } \\
\text { eyelid margin }\end{array}$ & $\mathrm{T} 2 \mathrm{a}$ & Tumour does not invade tarsal plate or eyelid margin & $\begin{array}{l}\text { are upgraded to } \mathrm{T} 2 \mathrm{a} \text { and } \mathrm{T} 2 \mathrm{~b} \text {, respectively, in the } 7 \text { th } \\
\text { edition, but the same features only affected the } \\
\text { classification of subcategory (e.g. a, b, c) within the same } \\
\mathrm{T} \text { category in the new system }\end{array}$ \\
\hline \multirow[t]{2}{*}{$\mathrm{T} 2 \mathrm{~b}$} & $\begin{array}{l}\text { Tumour }>10 \mathrm{~mm} \text {, but not }>20 \mathrm{~mm} \text {, or } \\
\text { involves full thickness eyelid }\end{array}$ & $\mathrm{T} 2 \mathrm{~b}$ & Tumour invade tarsal plate or eyelid margin & \\
\hline & & $\mathrm{T} 2 \mathrm{c}$ & Tumour invade full thickness of the eyelid & \\
\hline \multirow[t]{2}{*}{ T3a } & $\begin{array}{l}\text { Tumour }>20 \mathrm{~mm} \text {, or any tumour } \\
\text { invading adjacent ocular or orbital } \\
\text { structures; any } \mathrm{T} \text { with perineural } \\
\text { invasion }\end{array}$ & T3 & Tumour $>20 \mathrm{~mm}$ but $\leq 30 \mathrm{~mm}$ in greatest dimension & \\
\hline & & $\mathrm{T} 3 \mathrm{a}$ & Tumour does not invade tarsal plate or eyelid margin & \\
\hline \multirow[t]{2}{*}{$\mathrm{T} 3 \mathrm{~b}$} & $\begin{array}{l}\text { Complete tumour resection requires } \\
\text { enucleation, exenteration, or bone } \\
\text { resection }\end{array}$ & $\mathrm{T} 3 \mathrm{~b}$ & Tumour invade tarsal plate or eyelid margin & \\
\hline & & $\mathrm{T} 3 \mathrm{c}$ & Tumour invade full thickness of the eyelid & \\
\hline \multirow[t]{3}{*}{ T4 } & $\begin{array}{l}\text { Tumour is not resectable because of } \\
\text { extensive invasion of ocular, orbital, or } \\
\text { craniofacial structures or brain }\end{array}$ & $\mathrm{T} 4$ & $\begin{array}{l}\text { Eyelid tumour invading adjacent ocular, orbital, or } \\
\text { facial structures }\end{array}$ & \\
\hline & & $\mathrm{T} 4 \mathrm{a}$ & Tumour invades ocular or intraorbital structures & \\
\hline & & $\mathrm{T} 4 \mathrm{~b}$ & $\begin{array}{l}\text { Tumour invades(or erodes through) the bony walls of } \\
\text { the orbit or extends to the paranasal sinuses or invades } \\
\text { the lacrimal sac/nasolacrimal duct or brain }\end{array}$ & \\
\hline
\end{tabular}

patient distribution using the 7th and 8th editions of the AJCC T category. The performance of the two editions was evaluated for the outcomes of local recurrence, regional lymph node metastasis, and tumour-related death.

\section{Methods}

\section{Patients}

We retrospectively reviewed all consecutive cases with the histopathological diagnosis of eyelid SGC from January 2000 to September 2015 in National Taiwan University Hospital, treated by a single surgeon (S.-L.L.). Patients with missing data would be excluded. This study was approved by the Institutional Review Board at National Taiwan University Hospital and adhered to the tenets of the Declaration of Helsinki. A detailed chart review was conducted for clinical data that included general and demographic information, associated ocular and systemic diseases, history of local irradiation, and well-known risk factors including Muir-Torre syndrome, and immunocompromised status. Other recorded features included symptom duration, laterality, initial presentation, histopathological features, extent of tumour involvement, initial clinical and pathological diagnoses, modality of treatment, follow-up duration, evidence of local recurrence, regional lymph node metastasis or systemic metastasis, and time to the events.
Histopathological features such as tumour dimension, growth pattern, pagetoid spread, basaloid or squamoid differentiation, as well as perivascular, lymphatic, and perineural invasions were recorded. During the follow-up period, local recurrence and regional lymph node metastasis were monitored by physical examination. Excisional biopsy or fine needle aspiration of lymph nodes was performed if any suspicious lesion was noted. Regular head and neck MRI was performed in patient with advanced disease. The modality of primary treatment was also recorded. For patients who underwent re-treatment, the indications and details of treatment were documented. All patients were classified retrospectively based on the T category of the primary tumour according to both the 7th and 8th editions AJCC staging systems [12, 13].

\section{Statistical analysis}

All statistical analyses were performed using R (version 3.5.1). To identify the prognostic factors for local recurrence, regional lymph node metastasis, and tumour-related death, Cox proportional hazards regression with competing risks was performed [14]. For local recurrence and regional lymph node metastasis, both tumour and nontumour-related deaths were considered as competing events. For tumour-related death, non-tumour-related deaths were considered as competing events. Schoenfeld residuals were obtained to test the proportional hazard 
assumptions of Cox regressions. A $P$-value of $<0.05$ was considered to be statistically significant.

\section{Performance analysis of two staging systems}

The performance and predictive value of the 7 th and 8 th editions AJCC staging systems were assessed by the following criteria: [15] (1) patients with the same $\mathrm{T}$ stage were expected to have similar survival time, was supported if Akaike information criterion was low and Harrell's C index was high. For each staging system, Cox proportional hazards regression on T-stage, adjusted with gender and age on diagnosis was implemented to obtain the Akaike information criterion and Harrell's c-index; (2) patients in different Tstages were expected to have greater differences in survival. Kaplan-Meier analyses were implemented to study the effect of different T-stages on survival. Log-rank tests with a significance level of 0.05 were performed for comparing the survival of different T-stage groups; and (3) the survival of patients in earlier stages was better than the survival of patients in more advanced stages in a good prognostic system, which was defined as monotonicity of gradients. The ordinal relationship in survival time between stages was observed from the Kaplan-Meier survival curves.

\section{Results}

\section{Demographics and clinicopathological characteristics}

We retrospectively identified 72 eyelid SGC patients, nine patients were excluded due to missing data. Of 63 patients included in this study, 38 were women and 25 were men. The median age was 71 years (range 38-94 years). The median duration of symptoms was 12 months (range 0.25-240 months). Upper eyelid was the most common location (41\%), followed by lower eyelid (38\%) and both eyelids (21\%). Most cases presented as nodular masses, and the median tumour dimension was $11 \mathrm{~mm}$ (range 2-46 mm). Pagetoid spread was discovered in 23 cases (37\%). The most common pattern of tumour growth was lobular (64\%). The detailed clincopathological features are summarised in Table 2.

Most patients (91\%) underwent wide excision under frozen section control and simultaneous eyelid reconstruction as primary treatment, while six patients $(10 \%)$ had primary exenteration. Seven patients $(11 \%)$ having positive surgical margins refused exenteration or further surgery and underwent radiotherapy. Fifteen patients $(24 \%)$ developed local recurrence at a median interval of 16 months (range 5-346 months) during a median follow-up period of 46 months (range 1-362 months). Patients with local recurrence received wide excision $(n=9)$ or exenteration $(n=7)$.
Table 2 Clinical presentations and histopathological features

\begin{tabular}{|c|c|}
\hline \multicolumn{2}{|l|}{ Demographics } \\
\hline Male gender & $25(40)$ \\
\hline Age (years) & $71(38-94)$ \\
\hline \multicolumn{2}{|l|}{ Tumour extent } \\
\hline Upper eyelid & $26(41)$ \\
\hline Lower eyelid & $24(38)$ \\
\hline Conjunctiva & $16(25)$ \\
\hline Both eyelids & $13(21)$ \\
\hline Orbit & $11(18)$ \\
\hline Paranasal sinus & $4(6)$ \\
\hline Intracranium & $3(5)$ \\
\hline Caruncle & $2(3)$ \\
\hline Orbital wall & $2(3)$ \\
\hline Lacrimal system & $2(3)$ \\
\hline Nasal cavity & $1(2)$ \\
\hline \multicolumn{2}{|l|}{ Presenting sign } \\
\hline Nodular & $56(89)$ \\
\hline Lid thickening & $5(8)$ \\
\hline Nodular mass + Lid thickening & $2(3)$ \\
\hline \multicolumn{2}{|l|}{ Histopathological features } \\
\hline Pagetoid spread & $23(37)$ \\
\hline Basaloid differentiation & $22(35)$ \\
\hline Squamoid differentiation & $11(18)$ \\
\hline Lymphatic invasion & $6(10)$ \\
\hline Perivascular invasion & $5(8)$ \\
\hline Perineural invasion & $4(6)$ \\
\hline \multicolumn{2}{|l|}{ Section margin } \\
\hline Free & $40(64)$ \\
\hline Very close & $7(11)$ \\
\hline Not free & $16(25)$ \\
\hline \multicolumn{2}{|l|}{ Tumour growth pattern } \\
\hline Comedo & $20(32)$ \\
\hline Lobular & $40(64)$ \\
\hline Papillary & $1(2)$ \\
\hline Mixed & $2(3)$ \\
\hline
\end{tabular}

$N(\%)$; median (range)

Fourteen patients (22\%) developed regional lymph node metastasis at a median interval of 14 months (range $1-120$ months), mostly in the parotid region (19\%), followed by the submandibular region (3\%) and the neck (3\%). Patients with regional lymph node metastasis were treated with lymphadenectomy $(n=7)$ and radiotherapy $(n=13)$. Tumourrelated death occurred in four patients $(6 \%)$ with a median interval of 80.5 months (range 20-362 months), including three patients with intracranial extension and one with systemic metastasis involving the liver, brain, and lung diagnosed at the 16th month. Three patients $(4.8 \%)$ had nontumour-related death. 


\section{Prognostic factors}

Local recurrence was associated with perineural invasion, perivascular invasion, lymphatic invasion, basaloid differentiation, pagetoid spread, involvement of both eyelids, paranasal sinus, intracranium, and orbit. Clear surgical margins was a protective factor against local recurrence (Table 3) (Cox proportional hazards regression with competing risks).

Regional lymph node metastasis was associated with tumour dimension $>20 \mathrm{~mm}$, paranasal sinus involvement, orbital involvement, and positive surgical margins (Table 3) (Cox proportional hazards regression with competing risks). The prognostic factors for systemic metastasis could not be analysed because there was only one such case.

Tumour-related death had associations with perineural invasion and sinus involvement (Table 3) (Cox proportional hazards regression with competing risks). The results of intracranial extension and positive surgical margins were not available due to limited event numbers. All patients with intracranial extension and $25 \%$ of patients with positive surgical margins died of SGC.

\section{Comparison of 7th and 8th edition AJCC staging system}

The classification of patients according to the $\mathrm{T}$ categories of the 7th and 8th editions AJCC staging system is shown in Table 4. Thirty-five (55.6\%) patients remained in the same stage. Eight $(12.7 \%)$ patients were upgraded, while 20 (31.7\%) patients were downgraded in the 8th edition. All patients with $\mathrm{T} 1$ and $\mathrm{T} 2 \mathrm{a}$ tumours in 7 th edition were reclassified as $\mathrm{T} 1 \mathrm{a}$ and $\mathrm{T} 1 \mathrm{~b}$ in the 8th edition, respectively. Most (83\%) of the T2b tumours in the 7th edition AJCC staging system remained in T2b subgroup in the 8th edition AJCC staging system. The T3 tumours defined by the 7th edition AJCC staging system were the most affected group by the switch and had diverse classifications using the 8th edition AJCC staging system. Two cases originally classified as T3a due to perineural invasion were downgraded to $\mathrm{T} 2$, while three cases with orbital involvement were upgraded to T4. Similar condition was noted in T3b of 7th edition, which was based on tumour resectability. One of the T3b tumours only reached the criteria of T2c in 8th edition AJCC staging system, while five were reclassified as T4.

Performance analysis showed that the T category of 8th edition AJCC staging system had better predictive value for local recurrence and regional lymph node metastasis, supported by lower AIC values and higher or equal Harrell's cindices. The AIC value of 7 th edition and 8th edition for local recurrence was 108.2 and 106.6, respectively. The Harrell's c-index of 7th edition and 8th edition for local recurrence was 0.62 and 0.67 , respectively. The AIC value of 7th edition and 8th edition for regional lymph node metastasis was 157.7 and 94.6, respectively. The Harrell's c-index of 7th edition and 8th edition for regional lymph node metastasis were both 0.79 . Therefore, patients had the same $\mathrm{T}$ stage per 8th edition had more similar event-free

Table 3 Prognostic factors for local recurrence, regional lymph node metastasis, and tumour-related death

\begin{tabular}{|c|c|c|c|c|c|c|}
\hline & \multicolumn{2}{|l|}{ Local recurrence } & \multicolumn{2}{|c|}{ Regional lymph node metastasis } & \multicolumn{2}{|l|}{ Tumour-related death } \\
\hline & Hazard ratio $(95 \% \mathrm{CI})$ & $p$-value & Hazard ratio $(95 \% \mathrm{CI})$ & $p$-value & Hazard ratio $(95 \% \mathrm{CI})$ & $p$-value \\
\hline Age $>50$ (years) & $0.49(0.11-2.11)$ & 0.335 & $0.42(0.13-1.35)$ & 0.232 & $0.23(0.02-2.49)$ & 0.227 \\
\hline Gender (male) & $1.01(0.35-2.9)$ & 0.985 & $2.07(0.72-5.99)$ & 0.179 & $0.74(0.08-7.05)$ & 0.797 \\
\hline \multicolumn{7}{|l|}{ Histopathology } \\
\hline Tumour dimension $>20 \mathrm{~mm}$ & $1.08(0.3-3.89)$ & 0.902 & $3.07(1.02-9.21)$ & 0.045 & $2.66(0.23-31.03)$ & 0.435 \\
\hline Perineural invasion & $3.85(1.06-13.97)$ & 0.04 & $1.11(0.14-8.46)$ & 0.923 & $19.63(2.66-144.71)$ & 0.003 \\
\hline Perivascular invasion & $8.18(2.37-28.3)$ & 0.001 & $1.6(0.36-7.21)$ & 0.538 & $5.06(0.46-55.94)$ & 0.186 \\
\hline Lymphatic invasion & $5.23(1.59-17.26)$ & 0.007 & $1.3(0.29-5.88)$ & 0.73 & $4.22(0.38-47.05)$ & 0.241 \\
\hline Basaloid differentiation & $3.62(1.23-10.6)$ & 0.019 & $1.21(0.42-3.5)$ & 0.726 & $10.68(0.82-138.94)$ & 0.07 \\
\hline Squamoid differentiation & $1.03(0.29-3.69)$ & 0.965 & $0.64(0.14-2.89)$ & 0.566 & $6.84(0.62-75.67)$ & 0.117 \\
\hline Pagetoid spread & $5.47(1.6-18.72)$ & 0.007 & $1.55(0.54-4.44)$ & 0.414 & $3.07(0.35-27.16)$ & 0.313 \\
\hline Section margin free & $0.12(0.03-0.44)$ & 0.001 & $0.23(0.07-0.72)$ & 0.012 & Not available ${ }^{a}$ & \\
\hline \multicolumn{7}{|l|}{ Tumour involvement } \\
\hline Both eyelids & $4.71(1.63-13.62)$ & 0.004 & $1.63(0.51-5.21)$ & 0.409 & $7.82(0.7-87.13)$ & 0.095 \\
\hline Paranasal sinus & $4.85(1.35-17.41)$ & 0.016 & $5.09(1.39-18.56)$ & 0.014 & $17.5(1.55-197.38)$ & 0.021 \\
\hline Intracranium & $11.26(2.79-45.34)$ & 0.001 & $3.33(0.74-14.96)$ & 0.117 & Not available $^{\mathrm{a}}$ & \\
\hline Orbit & $3.84(1.33-11.08)$ & 0.016 & $3.31(1.1-9.95)$ & 0.033 & $8.43(0.77-92.04)$ & 0.08 \\
\hline Orbital wall & $1.93(0.27-13.58)$ & 0.508 & $2.66(0.34-20.51)$ & 0.348 & $0.75(0.01-37.94)$ & 0.886 \\
\hline
\end{tabular}

${ }^{a}$ Number of events is too small to perform Cox regression analyses 
Table 4 The distribution of patients in the 7th edition and the 8th edition AJCC staging system

\begin{tabular}{|c|c|c|c|c|c|c|c|c|c|c|c|c|c|c|}
\hline \multirow[b]{2}{*}{7 th } & \multirow[t]{2}{*}{8 th } & \multicolumn{3}{|l|}{$\mathrm{T} 1$} & \multicolumn{3}{|l|}{$\mathrm{T} 2$} & \multicolumn{3}{|l|}{$\mathrm{T} 3$} & \multicolumn{2}{|l|}{$\mathrm{T} 4$} & \multicolumn{2}{|c|}{ Total } \\
\hline & & $\mathrm{T} 1 \mathrm{a}$ & $\mathrm{T} 1 \mathrm{~b}$ & $\mathrm{~T} 1 \mathrm{c}$ & $\mathrm{T} 2 \mathrm{a}$ & $\mathrm{T} 2 \mathrm{~b}$ & $\mathrm{~T} 2 \mathrm{c}$ & $\mathrm{T} 3 \mathrm{a}$ & $\mathrm{T} 3 \mathrm{~b}$ & $\mathrm{~T} 3 \mathrm{c}$ & $\mathrm{T} 4 \mathrm{a}$ & $\mathrm{T} 4 \mathrm{~b}$ & & \\
\hline $\mathrm{T} 1$ & $\mathrm{~T} 1$ & 6 & 0 & 0 & 0 & 0 & 0 & 0 & 0 & 0 & 0 & 0 & 6 & $6(10 \%)$ \\
\hline \multirow[t]{2}{*}{$\mathrm{T} 2$} & $\mathrm{~T} 2 \mathrm{a}$ & 0 & 16 & 0 & 0 & 0 & 0 & 0 & 0 & 0 & 0 & 0 & 16 & $40(63 \%)$ \\
\hline & $\mathrm{T} 2 \mathrm{~b}$ & 0 & 0 & 1 & 0 & 20 & 3 & 0 & 0 & 0 & 0 & 0 & 24 & \\
\hline \multirow[t]{2}{*}{$\mathrm{T} 3$} & $\mathrm{~T} 3 \mathrm{a}$ & 0 & 0 & 0 & 0 & 1 & 1 & 0 & 2 & 1 & 3 & 0 & 8 & $16(25 \%)$ \\
\hline & $\mathrm{T} 3 \mathrm{~b}$ & 0 & 0 & 0 & 0 & 0 & 1 & 0 & 0 & 2 & 4 & 1 & 8 & \\
\hline $\mathrm{T} 4$ & $\mathrm{~T} 4$ & 0 & 0 & 0 & 0 & 0 & 0 & 0 & 0 & 0 & 0 & 1 & 1 & $1(2 \%)$ \\
\hline \multirow[t]{2}{*}{ Total } & & 6 & 16 & 1 & 0 & 21 & 5 & 0 & 2 & 3 & 7 & 2 & 63 & $00 \%)$ \\
\hline & & \multicolumn{3}{|c|}{$23(37 \%)$} & \multicolumn{3}{|c|}{$26(41 \%)$} & \multicolumn{3}{|c|}{$5(8 \%)$} & \multicolumn{2}{|c|}{$9(14 \%)$} & & \\
\hline
\end{tabular}

survival (EFS) for local recurrence and regional lymph node metastasis. On the other hand, the $\mathrm{T}$ category of the 7 th edition AJCC staging system had better monotonicity of gradients, which indicated the survival of patients in earlier stages was better than the survival of patients in more advanced stages. Determined by the 8th edition, the Kaplan-Meier curves of local recurrence-free survival of T3 was superior to T1 and T2. Also, the Kaplan-Meier curves of regional lymph node metastasis-free survival crossed (T1 and T2; T3 and T4) (Fig. 1). In addition, the 7th edition performed slightly better than the 8th edition regarding local recurrence as indicated by the inter-stage log-rank tests. Kaplan-Meier analysis showed that the EFS for local recurrence was significantly worse for T4 tumours in the 7 th edition (T1/T2/T3 vs. T4, $p=0.035$, log-rank test), while no significant difference in EFS was detected in the 8th edition. The EFS of regional lymph node metastasis was significantly worse in patients with tumours T3a or worse (T1/T2 vs. T3/T4, $p=0.002$, log-rank test) in the 7 th edition, and in those with tumours T2c or worse $(\mathrm{T} 1 / \mathrm{T} 2 \mathrm{a} / \mathrm{T} 2 \mathrm{~b}$ vs. T2c/T3/T4, $p=0.002$, log-rank test) in the 8th edition. The EFS of tumour-related death were significantly worse in patients with tumours $\mathrm{T} 3 \mathrm{~b}$ or worse in both staging systems (7th: T1/T2/T3a vs. T3b/T4; 8th: T1/T2/T3a vs. $\mathrm{T} 3 \mathrm{bc} / \mathrm{T} 4, p=0.004$, log-rank test).

\section{Discussion}

The present study analysed the clinicopathological features of SGC in the ethnic Chinese population in Taiwan, as well as the prognostic factors for local recurrence, regional lymph node metastasis, and tumour-related death. This study validated the recently published $\mathrm{T}$ category of the 8th edition AJCC staging system and compared it with the 7 th edition. Using the 8th edition staging system, we showed that, in our study population, tumours classified as T2c or worse had higher risks of regional lymph node metastasis, while tumours $\mathrm{T} 3 \mathrm{~b}$ or worse were associated with more tumour-related death. The $\mathrm{T}$ category of the 8 th edition had better predictive value for local recurrence and regional lymph node metastasis but failed to preserve the monotonicity of gradients as compared with the T category of 7 th edition AJCC staging system.

Our cohort exhibited slight female predominance (femaleto-male ratio 1.52) and older age (71 years in median) that were consistent with previous research (e.g. female-to-male ratio 1.7-2.8, mean age 60-69 years) [16]. In contrast to the higher prevalence of diffuse eyelid thickening in Caucasians, most patients had nodular lesions in the present study, which confirmed the tendency for nodular presentation in Asian patients as reported by Watanabe et al. [10]. Local recurrence rate and regional lymph node metastasis rate were $24 \%$ and $22 \%$, respectively, which were comparable to those in the literature $[11,16]$. Our cohort had a relatively low prevalence of systemic metastasis $(2 \%)$ and tumour-related death $(6 \%)$ as compared with $8-67 \%$ and $3-41 \%$, respectively, as reported by previous studies [11]. The prognosis of eyelid SGC is improving gradually [2], contributing to the advanced knowledge of disease, prompt diagnosis and treatment. It is worth mentioning that three patients $(4.8 \%)$ in our cohort had intracranial extension, which was a very rare presentation of eyelid SGC. Hayashi et al. reported only three cases after reviewing the literature [17], while a recent study reported only one of 191 patients $(0.5 \%)$ in an Indian cohort [11]. All our patients died of locally invasive disease. Two of them had T4 tumour at initial presentation, while one patient with $\mathrm{T} 2 \mathrm{~b}$ tumour experienced multiple local recurrences under regular follow-up and developed intracranial extension. Racial difference may lead to variable clinical outcome. Further study could focus on the diverse clinical features between different racial groups and its clinical impact.

The prognostic factors for recurrence and metastasis were widely discussed in previous research [8, 18, 19]. We further specified outcomes as local recurrence, regional lymph node metastasis, and tumour-related death. The discussion of prognostic factors for individual outcomes is as follows.

Local recurrence tended to be associated with more aggressive histopathological patterns (e.g. perineural, perivascular, lymphatic invasion) and more extensive tumour 
$7^{\text {th }}$ edition
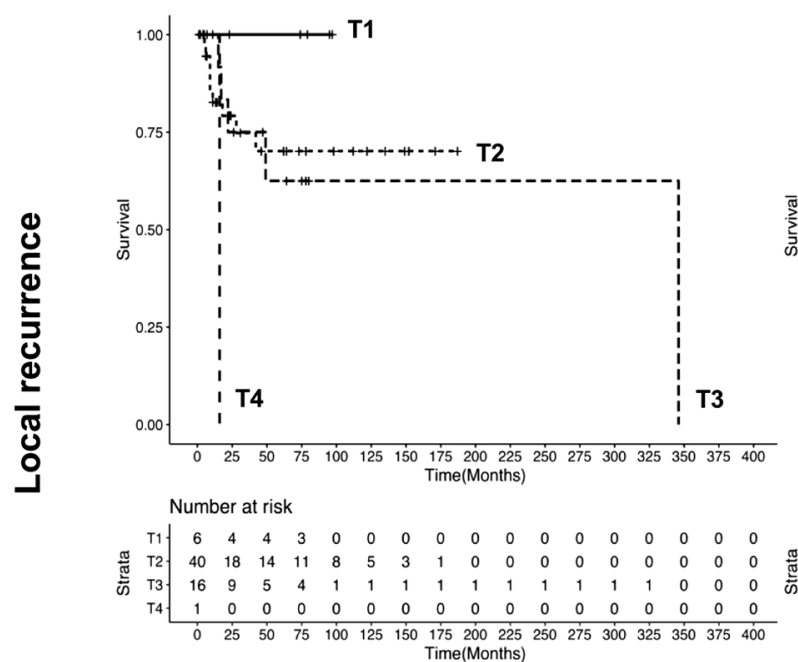
Time(Months)
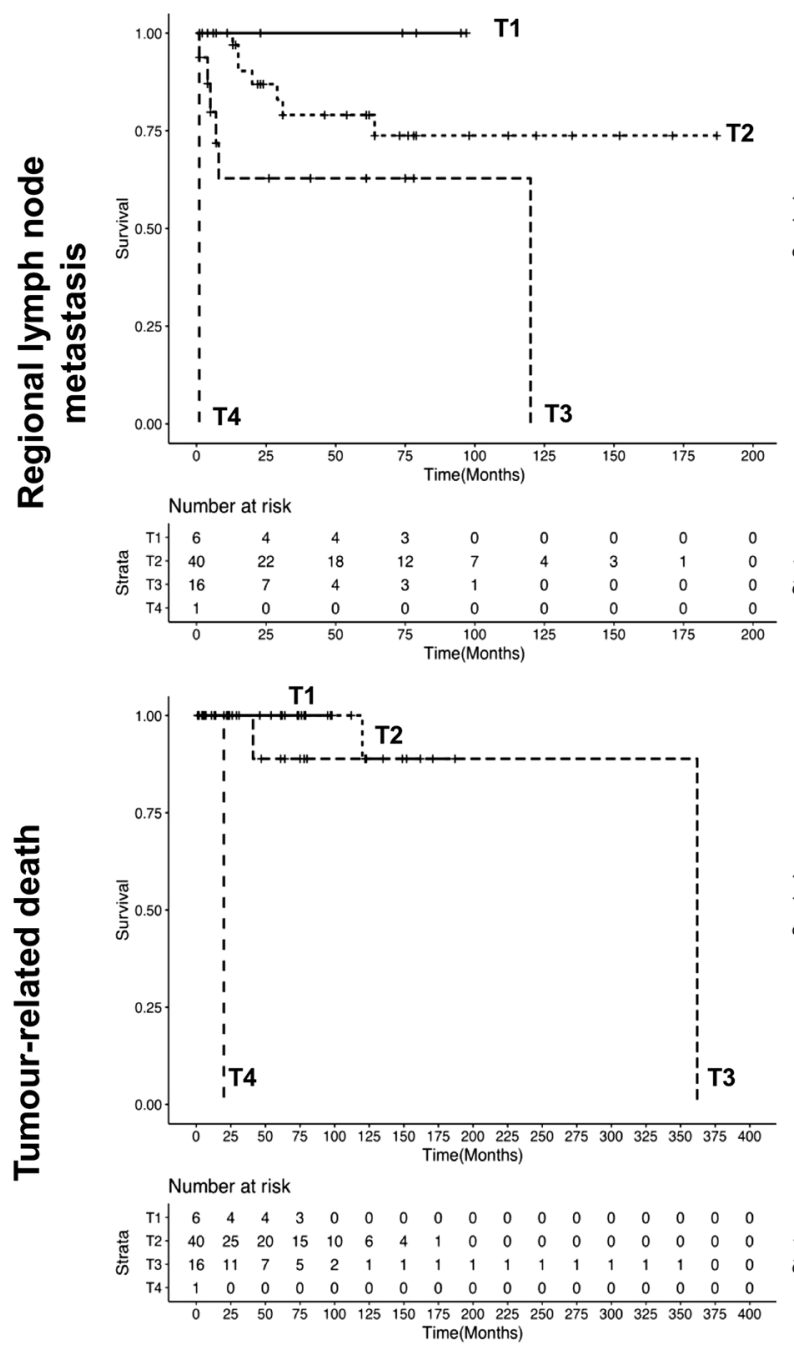

Fig. 1 The Kaplan-Meier survival curves for outcomes including local recurrence, regional lymph node metastasis, and tumour-related death. The patients were stratified according to $\mathrm{T}$ category of the 7 th or 8 th AJCC staging systems. The monotonicity of gradients can be observed

\section{$8^{\text {th }}$ edition}

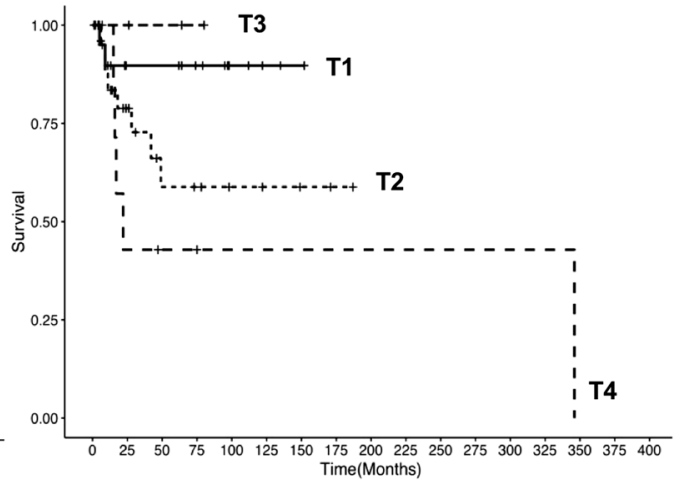

Number at risk

T1. $\begin{array}{lllllllllllllllll}23 & 11 & 11 & 8 & 4 & 2 & 1 & 0 & 0 & 0 & 0 & 0 & 0 & 0 & 0 & 0 & 0\end{array}$

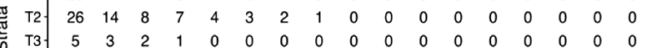

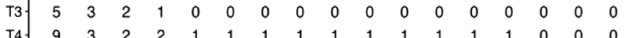

\begin{tabular}{ccccccccccccccccc}
9 & 3 & 2 & 2 & 1 & 1 & 1 & 1 & 1 & 1 & 1 & 1 & 1 & 1 & 0 & 0 & 0 \\
\hline 0 & 25 & 50 & 75 & 100 & 125 & 150 & 175 & 200 & 225 & 250 & 275 & 300 & 325 & 350 & 375 & 400
\end{tabular} Time(Months)
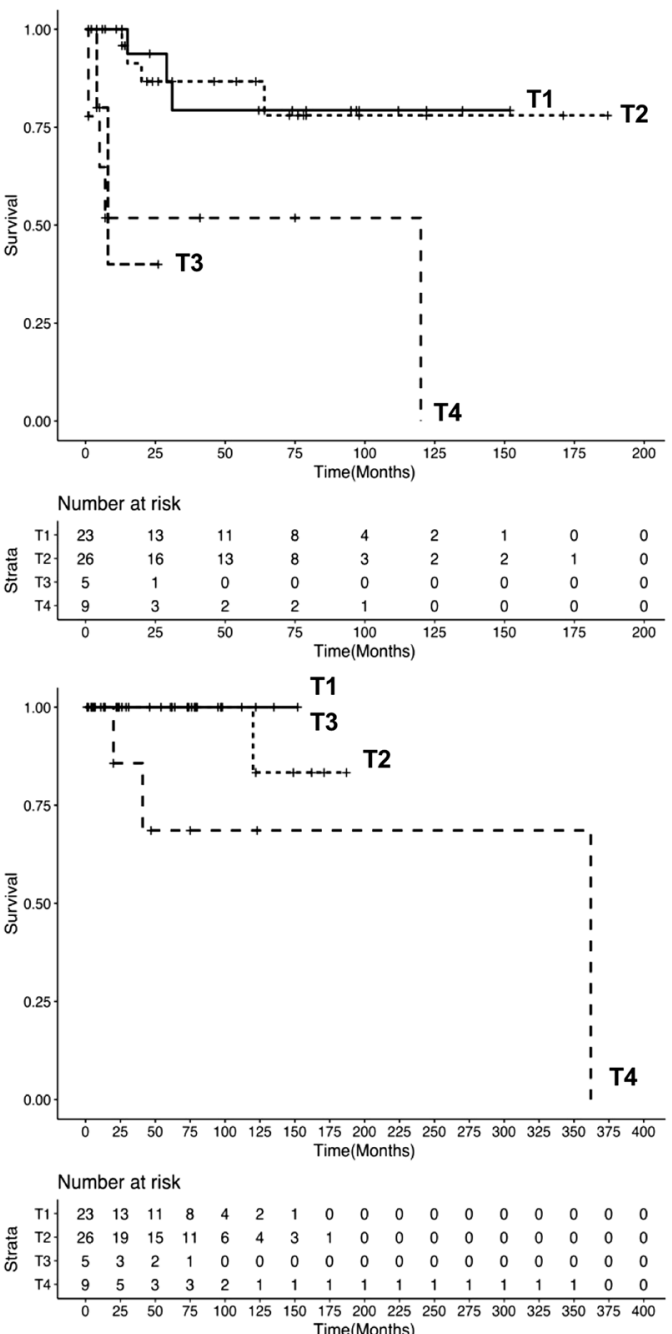

on the Kaplan-Meier survival curves. For the 8th edition staging system, the monotonicity of gradients of local recurrence, regional lymph node metastasis, and tumour-related death were not wellassociated with the severity of the stages 
invasion to the surroundings but not to tumour size. Basaloid and squamoid metaplasia are variants of SGC. We found local recurrence was associated with basaloid metaplasia but not with squamoid metaplasia. These findings suggested that patients with specific histopathological patterns should have close follow-ups. Patients with extensive tumour involvement should be closely scrutinised for local recurrence. Extensive resection or exenteration can be considered to prevent locally advanced disease.

SGC has been reported to have a high rate of regional lymph node metastasis among all the eyelid malignancies [20]. Therefore, it is important to identify the group at higher risk of regional lymph node metastasis and consider regular imaging follow-up and sentinel lymph node biopsy. The result in the literatures was conflicting in the association between tumour size and lymph node metastasis. In our study, the patients having tumour dimension $>20 \mathrm{~mm}$ were at higher risk of regional lymph node metastasis. In contrast to local recurrence, regional lymph node metastasis was unrelated to aggressive histopathological patterns or extensive tumour invasion in the present study. In addition, previous study showed that lymphatics of all portion of eyelids drain into the preauricular nodes [21]. It was supported by the observation in the present study that $86 \%$ of regional lymph node metastasis occurred in the parotid region. Therefore, patients with larger tumour should be scrutinised for regional lymph node metastasis, especially at parotid region.

The AJCC staging system was updated regularly to determine the "high risk groups" for poor outcomes and to guide the clinical treatment. Only one AJCC staging system is provided for eyelid carcinoma, and no specified staging system for SGC is available. There were several studies that tested the ability of the 7th edition to predict the prognosis of patients with eyelid SGC. Esmaeli et al. reported that tumours of T3a or worse correlated with increased metastasis and tumour-related death, but was not associated with local recurrence [9]. In contrast, Watanabe et al. found that tumours of T3a or worse was associated with local recurrence but detected no correlation for lymph node metastasis. The relatively low incidence of lymph node metastasis $(8 \%)$ and local recurrence $(8 \%)$ may have reduced the statistical power [10]. Kaliki et al. found that higher $\mathrm{T}$ category was associated with poor outcomes except for local recurrence [11]. In the present study, we classified the cases with both the 7th and 8th editions of AJCC staging system $[12,13]$ and found that the updates in the 8th edition (Table 1) improved the predictive power for local recurrence and regional lymph node metastasis. However, the $\mathrm{T}$ category of the 8th edition staging system demonstrated worse monotonicity of gradients compared with the 7th edition for eyelid SGC. In the present study, local recurrence was unrelated to tumour size, while regional lymph node metastasis showed a positive correlation with tumour size. Monotonicity of gradients regarding the survival analysis of local recurrence might have been lost
(T3 had best outcome). Because the T3 tumours were predominantly classified by tumour size in the 8th edition, while the local structures invasiveness and perineural invasion were considered in the 7th edition. Loss of monotonicity of gradients for the survival analysis of regional lymph node metastasis was also noted (T4 had better outcome than T3), as T4 tumours were not inevitably larger than T3 tumours in the 8th edition. In addition, both staging systems were not effective in predicting local recurrence, which was consistent with previous reports $[9,11]$. Our results showed that T4 tumour in the 7th edition had more local recurrence (T1/T2/T3 vs. T4, $p=0.035)$, which likely had little clinical significance. Therefore, based on the current system, all patients should be monitored for local recurrence. Histopathological pattern and tumour invasion to adjacent structures were associated with local recurrence, but only the latter were included in the new staging system. Histopathological pattern may be reconsidered in the future refinement. On the other hand, because the correlation with tumour size, the inter-stage difference of regional lymph node metastasis was better demonstrated in both staging systems. According to the 8th edition, patients with tumour size $>20$ $\mathrm{mm}$ or tumour size $>10 \mathrm{~mm}$ with full thickness eyelid involvement (T3b or worse) should be monitored for regional lymph node metastasis.

There were some limitations in this study. First, the case number was limited owing to the rarity of eyelid SGC and this was especially true for cases with higher stage disease. There was only one patient (1.6\%) who had T4 tumour according to the 7th edition. Therefore, our data may fail to present the clinical behaviour of that group well. However, the number of T4 tumours was also limited in other cohorts $(0-4 \%)$ [9-11], which may be related to the strict criteria of the T4 tumour in the 7th staging system. Second, our median follow-up period was 46 months and some recurrence or metastasis might have occurred beyond this period. However, the follow-up time was not significantly different between the patients with or without events.

In conclusion, this study demonstrated the clinicopathological features of eyelid SGC in the ethnic Chinese population in Taiwan. Eyelid SGC in Asians tended to present as nodular mass. We identified that local recurrence was associated with aggressive histopathological patterns and extensive local invasions, while regional lymph node metastasis occurred in patients with larger tumours. Since positive surgical margin was strongly associated with poor outcomes, more aggressive local control by surgery or radiotherapy was recommended to high risk groups. Compared to the 7th edition, the $\mathrm{T}$ category of the 8th edition of AJCC staging system demonstrated improved performance regarding local recurrence and regional lymph node metastasis in our cohort. Further studies to refine the T category of AJCC staging system are needed to improve the monotonicity of gradients between groups, and 
the association with histopathological patterns can be considered to improve the predictive value for local recurrence.

\section{Summary}

\section{What was known before}

- The performance of the 7th edition T category of AJCC staging system in eyelid sebaceous carcinoma.

\section{What this study adds}

- The clinical features and prognostic factors for eyelid SGC in Chinese population.

- The performance of the 8th edition T category of AJCC staging system in eyelid sebaceous carcinoma.

Acknowledgements English editing and statistical consulting were provided by the Department of Medical Research at National Taiwan University Hospital.

\section{Compliance with ethical standards}

Conflict of interest The authors declare that they have no conflict of interest.

Publisher's note: Springer Nature remains neutral with regard to jurisdictional claims in published maps and institutional affiliations.

\section{References}

1. Dasgupta T, Wilson LD, Yu JB. A retrospective review of 1349 cases of sebaceous carcinoma. Cancer. 2009;115:158-65.

2. Shields JA, Demirci H, Marr BP, Eagle RC Jr., Shields CL. Sebaceous carcinoma of the eyelids: personal experience with 60 cases. Ophthalmology. 2004;111:2151-7.

3. Silverman N, Shinder R. What's new in eyelid tumours. Asia Pac J Ophthalmol. 2017;6:143-52.

4. Wang JK, Liao SL, Jou JR, Lai PC, Kao SC, Hou PK, et al. Malignant eyelid tumours in Taiwan. Eye. 2003;17:216-20.

5. Sihota R, Tandon K, Betharia SM, Arora R. Malignant eyelid tumours in an Indian population. Arch Ophthalmol. 1996;114: $108-9$.

6. Takamura H, Yamashita H. Clinicopathological analysis of malignant eyelid tumour cases at Yamagata university hospital: statistical comparison of tumour incidence in Japan and in other countries. Jpn J Ophthalmol. 2005;49:349-54.

7. Wang CJ, Zhang HN, Wu H, Shi X, Xie JJ, He JJ, et al. Clinicopathologic features and prognostic factors of malignant eyelid tumours. Int J Ophthalmol. 2013;6:442-7.

8. Kaliki S, Ayyar A, Dave TV, Ali MJ, Mishra DK, Naik MN. Sebaceous gland carcinoma of the eyelid: clinicopathological features and outcome in Asian Indians. Eye. 2015;29:958-63.

9. Esmaeli B, Nasser QJ, Cruz H, Fellman M, Warneke CL, Ivan D. American Joint Committee on Cancer $\mathrm{T}$ category for eyelid sebaceous carcinoma correlates with nodal metastasis and survival. Ophthalmology. 2012;119:1078-82.

10. Watanabe A, Sun MT, Pirbhai A, Ueda K, Katori N, Selva D. Sebaceous carcinoma in Japanese patients: clinical presentation, staging and outcomes. $\mathrm{Br}$ J Ophthalmol. 2013;97: 1459-63.

11. Kaliki S, Gupta A, Ali MH, Ayyar A, Naik MN. Prognosis of eyelid sebaceous gland carcinoma based on the tumour $(\mathrm{T})$ category of the American Joint Committee on Cancer (AJCC) classification. Int J Ophthalmol. 2016;36:681-90.

12. Edge S, Byrd DR, Compton CC, Fritz AG, Greene FL, Trotti A, editors. AJCC cancer staging manual. 7th ed. New York: Springer; 2010.

13. Amin MB, Edge SB, Greene FL, Byrd DR, Brookland RK, Washington MK, et al., editors. AJCC cancer staging manual. 8th ed. New York: Springer; 2017.

14. Fine JP, Gray RJ. A proportional hazards model for the subdistribution of a competing risk. J Am Stat Assoc. 1999;94: 496-509.

15. Ueno S, Tanabe G, Sako K, Hiwaki T, Hokotate H, Fukukura Y, et al. Discrimination value of the new western prognostic system (CLIP score) for hepatocellular carcinoma in 662 Japanese patients. Cancer of the Liver Italian Program. Hepatology. 2001; 34:529-34.

16. Chao AN, Shields CL, Krema H, Shields JA. Outcome of patients with periocular sebaceous gland carcinoma with and without conjunctival intraepithelial invasion. Ophthalmology. 2001;108:1877-83.

17. Hayashi Y, Shima H, Kita D, Kinoshita M, Yoshida Y, Hasegawa $\mathrm{M}$, et al. Intracranial extension of meibomian gland carcinoma with pagetoid changes. J Clin Neurosci. 2009;16:568-70.

18. Rao NA, Hidayat AA, McLean IW, Zimmerman LE. Sebaceous carcinomas of the ocular adnexa: a clinicopathologic study of 104 cases, with five-year follow-up data. Hum Pathol. 1982;13:113-22.

19. Takahashi Y, Takahashi E, Nakakura S, Kitaguchi Y, Mupas-Uy J, Kakizaki H. Risk factors for local recurrence or metastasis of eyelid sebaceous gland carcinoma after wide excision with paraffin section control. Am J Ophthalmol. 2016;171:67-74.

20. Hashimoto K, Yasumatsu R, Toh S, Shiratsuchi H, Yoshida T, Nishiyama K, et al. Patterns of lymphatic spread and the management of eyelid carcinomas. Auris Nasus Larynx. 2016;43: 666-71.

21. Pfeiffer ML, Savar A, Esmaeli B. Sentinel lymph node biopsy for eyelid and conjunctival tumours: what have we learned in the past decade? Ophthal Plast Reconstr Surg. 2013;29:57-62. 